\title{
A Cyclic Prefix based Extended QRD-RLS Algorithm using Block Householder Transformation for Adaptive Channel Estimation in Multicarrier Systems
}

\author{
Hassan Ali
}

\begin{abstract}
This paper proposes a new cyclic prefix (CP) based extended QR decomposition Recursive Least Squares (QRD-RLS) algorithm using block Householder transformation (HT) for adaptive channel estimation in multicarrier systems. The new method is computationally cheaper than CP exploiting correlation matrix based block RLS (CMB-RLS) adaptive channel estimation approach, and possesses desirable properties for systolic array implementation. Simulation results suggest that as compared to the CMB-RLS scheme, the new algorithm possesses superior steady state performance.
\end{abstract}

Index Terms-Adaptive channel estimation, cyclic prefix, extended QRD-RLS, block householder transformation.

\section{INTRODUCTION}

Most multicarrier systems [1]-[3] use coherent detection of data symbols, which requires reliable estimation of channel at the receiver. Channel state information is also necessary for techniques such as channel shortening [4], adaptive modulation/loading and/or power control [5]. In applications such as discrete multi-tone (DMT) xDSL [6], channel is estimated through some initial training process and retraining is required to track the channel variation. To avoid the system overhead due to retraining and thus to track the channel more efficiently, in [7], a correlation matrix based block recursive least-squares (CMB-RLS) algorithm is proposed. The algorithm takes advantage of the inherent redundancy introduced by the cyclic prefix $(\mathrm{CP})$ to blindly estimate the channel. A basic problem with this approach is that it relies on computation of inverse of the correlation matrix $\overline{\boldsymbol{\Phi}}$ per time update, which requires $O\left(r^{3}\right)$ computations, where $r$ is the channel length. Obviously, in a real time system this cost can be prohibitively high with a large channel length.

In [8], Liu et al. investigated a $\mathrm{QR}$ decomposition recursive least squares (QRD-RLS) algorithm using block Householder transformation (HT). The work in [8] describes the block HT implementation on a systolic array and its application to RLS algorithm called systolic block HT RLS (SBHT-RLS). The problem with SBHT-RLS approach is that it does not provide access to channel weights, as its use has been limited to problem seeking an estimate of an output error signal.

In this paper, we propose a new $\mathrm{CP}$ based adaptive channel estimation algorithm using block HT for adaptive channel

Manuscript received June 10, 2012; revised July 11, 2012

Hassan Ali is with the School of Electrical Engineering and Computer Science, Faculty of Engineering and Built Environment, University of Newcastle, Australia (e-mail: hassan.ali@ newcastle.edu.au). estimation in multicarrier systems. The new method can be seen as an extension of Liu's method to find the channel explicitly through a time updating formula. The derivation of the channel updating in the new method is done by generalizing the extended QRD-RLS algorithm [9] to block RLS case. For this reason, this method will be referred to as $\mathrm{CP}$ based extended QRD-RLS algorithm. As compared to the CMB-RLS approach, the proposed arrangement is not only computationally efficient and amenable to systolic processors but also results in superior steady state performance.

In the rest of this paper, for clarity of presentation and uniformity, we closely follow the notation that appears in [7].

\section{SySTEM MODEL}

We consider a high speed DMT data transmission system over digital subscriber lines having $m / 2$ complex parallel subchannels. Let $\left\{s_{k}\right\}$ represent the data sequence to be transmitted over the channel. This input data is buffered to blocks, each data block is divided into $m / 2$ bit streams and then mapped to quadrature amplitude modulation (QAM) constellation points $X_{i, k}, i=0, \cdots, m / 2-1$ at time $k$. After $m$-point inverse fast Fourier transform (IFFT) on the $k$ th DMT block $\mathbf{X}_{k}=\left[X_{0, k}, X_{1, k}, \ldots, X_{m-1, k}\right]^{T}$ (here the last $m / 2$ samples are just the conjugates of the first $m / 2$ samples), the modulated real valued time domain signal is $\mathbf{x}_{k}=\left[x_{0, k}, x_{1, k}, \ldots, x_{m-1, k}\right]^{T}$. A CP $\quad \mathbf{x}^{(f)}{ }_{k}=\left[x_{-v, k}, \ldots, x_{-1, k}\right]^{T}$ (where $x_{-i, k}=x_{m-i, k}$ and $i=1, \ldots, v$ ) is then appended in front of $\mathbf{x}_{k}$ before transmission through the channel having impulse response $\mathbf{h}=\left[h_{0}, h_{1}, \ldots, h_{v}\right]^{T}$ of length $r=v+1$. At the receiver, the prefix part $\mathbf{y}_{k}^{(f)}=\left[y_{-v, k}, \ldots, y_{-1, k}\right]^{T}$ is removed.

The relationship between prefix part $\mathbf{y}_{k}^{(f)}$ and the transmitted signal may be expressed as [7]:

$$
\mathbf{y}_{k}^{(f)}=\mathbf{A}_{k} \mathbf{h}_{k}+\mathbf{n}_{k}^{(f)},
$$

where

$$
\mathbf{A}_{k}=\left[\begin{array}{cccc}
x_{-v, k} & x_{m-1, k-1} & \ldots & x_{m-v, k-1} \\
\ddots & \ddots & \ddots & \vdots \\
\ddots & \ddots & \ddots & \vdots \\
x_{-1, k} & \cdots & x_{-v, k} & x_{m-1, k-1}
\end{array}\right],
$$


$\mathbf{n}_{k}^{(f)}=\left[n_{-v, k}, \cdots, n_{-1, k}\right]^{T}$ and $n_{i, k} \sim N\left(0, \sigma^{2}\right)$ is the channel noise.

After the FFT operation on $\mathbf{y}_{k}=\left[y_{0, k}, y_{1, k}, \ldots, y_{m-1, k}\right]^{T}$, the demodulated signal is $\mathbf{Y}_{k}=\left[Y_{0, k}, Y_{1, k}, \ldots, Y_{m-1, k}\right]^{T}$. The $\mathrm{CP}$ removes inter-block-interference (IBI) between $\mathbf{X}_{k}{ }^{\prime} s$. The received symbols can thus be written as:

$$
Y_{i, k}=X_{i, k} H_{i}+N_{i, k}, \quad i=0, \ldots, m-1,
$$

where $H_{i}=\sum_{l=0}^{v} h_{l} e^{-j((2 \pi i l) / m)}$ is the channel frequency response and $N_{i, k}=(1 / \sqrt{m}) \sum_{l=0}^{m-1} n_{l} e^{-j((2 \pi i) / m)} \sim N\left(0, \sigma^{2}\right)$ is the noise of the $i$ th subchannel.

To get the estimation of $X_{i, k}$ from $Y_{i, k}$, a one tap minimum-mean-square-error

(MMSE) equalizer $w_{i}=\left(\Gamma_{i}^{1 / 2} H_{i}^{*}\right) /\left(\Gamma_{i}\left\|H_{i}\right\|^{2}+\sigma_{i}^{2}\right)($ where $i=0, \ldots, m-1$ and $\left.\Gamma_{i}=E\left[\left\|X_{i, k}\right\|^{2}\right]\right)$ is then employed at the $i$ th channel. The estimated data is then $\hat{X}_{i, k}=Y_{i, k} w_{i}$. The decision is then made on $\hat{X}_{i, k}$ to get the final output $\bar{X}_{i, k}=q\left(\hat{X}_{i, k}\right)$, where $q(\cdot)$ is the decision operation.

\section{CP BASED EXTENDED QRD-RLS ALGORITHM USING BLOCK HOUSEHOLDER TRANSFORMATION}

Based on the CP data model (1), we define $n v \times r$ weighted data matrix and the $n v \times 1$ weighted received vector in a recursive manner as

$$
\begin{gathered}
\dot{\mathbf{A}}_{k}=\Lambda\left[\begin{array}{c}
\mathbf{A}_{k-(n-1)} \\
\mathbf{A}_{k-n} \\
\vdots \\
\mathbf{A}_{k}
\end{array}\right]=\left[\frac{\lambda^{1 / 2} \dot{\mathbf{A}}_{k-1}}{\mathbf{A}_{k}}\right], \\
\dot{\mathbf{y}}_{k}^{(f)}=\Lambda\left[\begin{array}{c}
\mathbf{y}_{k-(n-1)}^{(f)} \\
\mathbf{y}_{k-n}^{(f)} \\
\vdots \\
\mathbf{y}_{k}^{(f)}
\end{array}\right]=\left[\frac{\lambda^{1 / 2} \dot{\mathbf{y}}_{k-1}^{(f)}}{\mathbf{y}_{k}^{(f)}}\right],
\end{gathered}
$$

where $\Lambda$ is an $n v \times n v$ block-diagonal forgetting matrix of the form

$$
\Lambda=\left[\begin{array}{cccc}
\lambda^{\frac{n-1}{2}} \mathbf{I}_{v} & \cdots & \mathbf{0} & \mathbf{0} \\
\vdots & \ddots & \vdots & \vdots \\
\mathbf{0} & \cdots & \lambda^{1 / 2} \mathbf{I}_{v} & \mathbf{0} \\
\mathbf{0} & \cdots & 0 & \mathbf{I}_{v}
\end{array}\right],
$$

with forgetting factor across blocks $0<\lambda \leq 1$. Suppose that at (k-1)th update we have QRD:

$$
\mathbf{Q}_{k-1} \dot{\mathbf{A}}_{k-1}=\left[\frac{\mathbf{R}_{k-1}}{\mathbf{0}}\right],
$$

where $\mathbf{Q}_{k-1}$ is an $(n-1) v \times(n-1) v$ orthogonal matrix and $\mathbf{R}_{k-1}$ is a $r \times r$ upper triangular matrix.

$$
\text { Now by denoting } \begin{gathered}
\overline{\mathbf{Q}}_{k-1}=\left[\begin{array}{cc}
\mathbf{Q}_{k-1} & \mathbf{0} \\
\mathbf{0}^{T} & \mathbf{I}_{v}
\end{array}\right], \text { we then have } \\
\overline{\mathbf{Q}}_{k-1} \dot{\mathbf{A}}_{k}=\left[\begin{array}{c}
\mathbf{R}_{k-1} \\
\mathbf{0} \\
\mathbf{A}_{k}
\end{array}\right] .
\end{gathered}
$$

A $n \times n$ Householder transformations matrix $\mathbf{T}$ is of the form $\mathbf{T}=\mathbf{I}_{n}-\beta \mathbf{v} \mathbf{v}^{T}$, where $\beta=2 / \mathbf{v}^{T} \mathbf{v}=2 /\|\mathbf{v}\|^{2}$. When a vector $\mathbf{x}=\left[x_{1}, x_{2}, \ldots, x_{n}\right]^{T}$ is multiplied by $\mathbf{T}$, it is reflected in the hyper plane defined by $\operatorname{span}\{\mathbf{v}\}^{\perp}$. Choosing $\mathbf{v}=\mathbf{x} \pm\|\mathbf{x}\|_{2} \mathbf{e}_{1}$, where $\mathbf{e}_{1}=[1,0,0, \ldots, 0]^{T}$, then $\mathbf{x}$ is reflected onto $\mathbf{e}_{1}$ by $\mathbf{T}$ as: $\mathbf{T x}= \pm\|\mathbf{x}\|_{2} \mathbf{e}_{1}$.

A series of Householder transformations are then used to zero out $\mathbf{A}_{k}$ in the right hand side of (7). Let $\mathbf{H}_{k}=\mathbf{H}_{k}^{(r)} \mathbf{H}_{k}^{(r-1)} \ldots \mathbf{H}_{k}^{(1)} \quad$ (a sequence of $r$-ordered matrix multiplications), where $\mathbf{H}_{k}^{(i)}$ denotes the $i$ th Householder transformation matrix (which zeros out $i$ th column of updated $\mathbf{A}_{k}$ ) given as

$$
\mathbf{H}_{k}^{(i)}=\left[\begin{array}{ccc}
\mathbf{H}_{k, 11}^{(i)} & \mathbf{0} & \mathbf{H}_{k, 12}^{(i)} \\
\mathbf{0} & \mathbf{I}_{(n-1) v-r} & \mathbf{0} \\
\mathbf{H}_{k, 21}^{(i)} & \mathbf{0} & \mathbf{H}_{k, 22}^{(i)}
\end{array}\right],
$$

where $\mathbf{H}_{k, 11}^{(i)}$ is $r \times r$ identity matrix except for the $i$ th diagonal entry; $\mathbf{H}_{k, 12}^{(i)}$ is $r \times v$ zero matrix except for the $i$ th row; $\mathbf{H}_{k, 12}^{(i)}=\mathbf{H}_{k, 21}^{(i)} ; \mathbf{H}_{k, 22}^{(i)}$ and is a symmetric $v \times v$ matrix.

It is thus we have $\mathbf{H}_{k} \overline{\mathbf{Q}}_{k-1} \dot{\mathbf{A}}_{k}=\left[\frac{\mathbf{R}_{k}}{\mathbf{0}}\right]$ and $\mathbf{Q}_{k}=\mathbf{H}_{k} \overline{\mathbf{Q}}_{k-1}$. Now with

$$
\mathbf{Q}_{k}\left[\begin{array}{ll}
\dot{\mathbf{A}}_{k} & \dot{\mathbf{y}}_{k}^{(f)}
\end{array}\right]=\left[\begin{array}{cc}
\mathbf{R}_{k} & \mathbf{u}_{k} \\
\mathbf{0} & \mathbf{v}_{k}
\end{array}\right]
$$

where $\mathbf{u}_{k}$ is $r \times 1$ and $\mathbf{v}_{k}$ is $(n v-r) \times 1$ vectors.

The matrix $\dot{\mathbf{A}}_{k-1}$ can be uniquely $\mathrm{QR}$ factorized only if it is full column rank (i.e., rank $\dot{\mathbf{A}}_{k-1}=r$ ). To satisfy this requirement the minimum number of rows in $\dot{\mathbf{A}}_{k-1}$ must be at least large as the number of columns. To satisfy this requirement and thus to reduce the number of received blocks needed, in Step (9) above, we set

$$
\dot{\mathbf{A}}_{k}=\left[\begin{array}{c}
\lambda^{1 / 2} R_{k-1} \\
\overline{\mathbf{A}}_{k}
\end{array}\right], \dot{\mathbf{y}}_{k}^{(f)}=\Lambda\left[\begin{array}{c}
\mathbf{y}_{k-2}^{(f)}(v)=y_{-1, k-2} \\
\mathbf{y}_{k-1}^{(f)} \\
\vdots \\
\mathbf{y}_{k}^{(f)}
\end{array}\right] .
$$

Now, by combining the first column of (9) and a new column, we construct the formula: 


$$
\mathbf{Q}_{k}\left[\begin{array}{cc}
\mathbf{R}_{k-1}^{-T} / \sqrt{\lambda} & \sqrt{\lambda} \mathbf{R}_{k-1} \\
\mathbf{0}^{T} & \mathbf{A}_{k}
\end{array}\right]=\left[\begin{array}{cc}
\mathbf{R}_{k}^{-T} & \mathbf{R}_{k} \\
\mathbf{U}_{k} & \mathbf{0}
\end{array}\right],
$$

We define a lemma, known as the matrix factorization lemma [10] that is very elegant tool in the development of QRD-RLS algorithms.

Lemma 1. If $\mathbf{A}$ and $\mathbf{B}$ are any two $N \times M(N \leq M)$ matrices, then

$$
\mathbf{A}^{T} \mathbf{A}=\mathbf{B}^{T} \mathbf{B} .
$$

if and only if there exists an $N \times N$ unitary matrix $\mathbf{Q}$ such that

$$
\mathbf{Q A}=\mathbf{B} .
$$

Applying Lemma 1 to (10), we obtain

$$
\mathbf{R}_{k}^{-T} \mathbf{R}_{k}^{T}=\mathbf{R}_{k-1}^{-T} \mathbf{R}_{k-1}^{T}+\mathbf{I}
$$

This shows that $\mathbf{R}_{k}^{-T}$ obtained is the correct inverse transposition of $\mathbf{R}_{k}^{T}$ and can be updated by using the same orthonormal transformation $\mathbf{Q}_{k}$.

Next, we combine the second column of (9) and the new column to construct the formula:

$$
\mathbf{Q}_{k}\left[\begin{array}{cc}
\mathbf{R}_{k-1}^{-T} / \sqrt{\lambda} & \sqrt{\lambda} \mathbf{y}_{k-1}^{(f)} \\
\mathbf{0}^{T} & \mathbf{y}_{k}^{(f)}
\end{array}\right]=\left[\begin{array}{cc}
\mathbf{R}_{k}^{-T} & \mathbf{u}_{k} \\
\mathbf{U}_{k}^{T} & \mathbf{v}_{k}
\end{array}\right] .
$$

Now by applying Lemma 1 to (14) yields

$$
\mathbf{R}_{k}^{-1} \mathbf{u}_{k}+\mathbf{U}_{k} \mathbf{v}_{k}=\mathbf{R}_{k-1}^{-1} \mathbf{u}_{k-1}
$$

From (15), we establish a simple recursion to compute the channel vector

$$
\mathbf{h}_{k}=\mathbf{h}_{k-1}-\mathbf{U}_{k} \mathbf{v}_{k} .
$$

Based upon the above discussion, the new algorithm is formulated in Table I. The algorithm is initialized in a training mode, the algorithm then switches to a decision directed mode for channel tracking. Note that, in Step 1, based on the previous channel estimate $\hat{\mathbf{h}}_{k-1}$, the previous frequency response $\tilde{H}_{k-1}=\left[H_{0, k-1}, H_{1, k-1}, \ldots, H_{m-1, k-1}\right]$ is computed. In Step 2, $\tilde{H}_{k-1}$ is then used to compute equalization coefficients. The decision directed data vector $\mathbf{X}_{k}$ is then computed in Step 3. In Step 4, symbol estimates are projected onto the finite alphabet (FA), and the estimated transmitted CP data $\overline{\mathbf{x}}^{(f)}{ }_{k}$ is obtained by performing partial FFT on the decision-directed projected samples $\overline{\mathbf{X}}_{k}$. In Step 5 through 7 , the new channel estimate is then obtained by the algorithm, by treating the resulting symbol estimates as the known symbols. The process of alternating between channel and symbol estimation steps is applied repeatedly.

The CP based CMB-RLS and extended QRD-RLS algorithms are similar in the $\mathrm{CP}$ estimation part (i.e., steps 1 through 4). The CMB-RLS channel estimation stage requires $O\left(r^{3}\right)$ computations to update $\mathbf{h}$. In contrast it is possible to implement extended QRD-RLS channel estimation part with $O\left(r^{2}\right)$ operations per time update. The proposed algorithm is thus computationally superior than the CMB-RLS algorithm.

The detection part of the proposed algorithm (comprising of Steps 1 through 4) is standard complaint for which many efficient systolic array architectures have been proposed. Adaptive filtering part (comprising of steps 5 through 7) can be easily realized on a rhombic systolic array to achieve massive parallel processing with vector operations.

TABLE I: CP BASED EXTENDED QRD-RLS ALGORITHM USING BLOCK HOUSEHOLDER TRANSFORMATIONS

Input: $\mathbf{y}_{k}^{(f)}, \mathbf{y}_{k-1}^{(f)}, \ldots \mathbf{y}_{k-2}^{(f)}(v)$ and $\mathbf{Y}_{k}$

Known parameters: $\Gamma_{i}$ and $\sigma_{i}$

Selecting parameters: $\lambda$ with $0<\lambda \leq 1$ )

Initialization: $k=0$, an initial training process is used to initialize $\hat{\mathbf{h}}$ $\mathbf{R}_{0}=\delta \mathbf{I}, \mathbf{R}_{0}^{-T}=\delta^{-1} \mathbf{I}$ with $0<\delta<<1$ is small positive scalar).

Algorithm: $k=1,2,3, \cdots$

1) $\mathcal{H}_{i, k-1}=(1 / \sqrt{m}) \sum_{l=0}^{v} \hat{h}_{l, k-1} e^{-j(2 \pi i l) / m}, i=0, \ldots, m-1$

2) $w_{i}=\left(\Gamma_{i}^{1 / 2} \mathcal{H}_{i}^{*}\right) /\left(\Gamma_{i}\left\|\mathcal{H}_{i}\right\|^{2}+\sigma_{i}^{2}\right), i=0, \ldots, m-1$

3) $\hat{X}_{i, k}=Y_{i, k} w_{i, k-1}, i=0, \ldots, m-1$

4) $\bar{x}_{i, k}=(1 / \sqrt{m}) \sum_{l=0}^{m-1} q\left(\hat{X}_{l, k}\right) e^{j(2 \pi i l) / m}, i=m-v, \ldots, m-$

5) $\dot{\mathbf{A}}_{k}=\left[\begin{array}{c}\sqrt{\lambda} \mathbf{R}_{k-1} \\ \overline{\mathbf{A}}_{k}\end{array}\right], \dot{\mathbf{B}}_{k}=\left[\begin{array}{c}\mathbf{R}_{k-1}^{-T} / \sqrt{\lambda} \\ 0^{T}\end{array}\right]$,

$\dot{\mathbf{y}}_{k}^{(f)}=\left[\begin{array}{c}\lambda \mathbf{y}_{k-2}^{(f)}(v) \\ \sqrt{\lambda} \mathbf{y}_{k-1}^{(f)} \\ \mathbf{y}_{k}^{(f)}\end{array}\right]=\left[\begin{array}{c}\sqrt{\lambda} \dot{\mathbf{y}}_{k-1}^{(f)} \\ \mathbf{y}_{k}^{(f)}\end{array}\right]$

6) $\mathbf{H}_{k}\left[\dot{\mathbf{A}}_{k}\left|\dot{\mathbf{B}}_{k}\right| \dot{\mathbf{y}}_{k}^{(f)}\right]=\left[\begin{array}{c|c|c}\mathbf{R}_{k} & \mathbf{R}_{k}^{-T} & \mathbf{u}_{k} \\ 0 & \mathbf{U}_{k}^{T} & \mathbf{v}_{k}\end{array}\right]$, where $\mathbf{H}_{k}=\mathbf{H}_{k}^{(r)} \mathbf{H}_{k}^{(r-1)}, \ldots, \mathbf{H}_{k}^{(1)}$,

with $\mathbf{H}_{k}^{(i)}=\mathbf{I}_{p-i+1}-\beta \mathbf{v} \mathbf{v}^{T}, p=2 v+1, \beta=2 / \mathbf{v}^{T} \mathbf{v}, \mathbf{v}=$ $\mathbf{x} \pm\|\mathbf{x}\|_{2} \mathbf{e}_{1}, \mathbf{e}_{1}=[1,0, \cdots, 0]^{T}$, and $\mathbf{x}=\dot{\mathbf{A}}_{k}(i: p, i)$.

7) $\mathbf{h}_{k}=\mathbf{h}_{k-1}-\mathbf{U}_{k} \mathbf{v}_{k}$

TABLE II: STATIC FIR CHANNEL MODELS

\begin{tabular}{c|c|c}
\hline \hline Tap \# & Channel A & Channel B \\
\hline 0 & 0.31220 & 0.53120 \\
\hline 1 & 0.05010 & 1.90100 \\
\hline 2 & 0.20560 & 0.00705 \\
\hline 3 & 0.27540 & 0.97540 \\
\hline 4 & 0.15670 & 0.01568 \\
\hline \hline
\end{tabular}
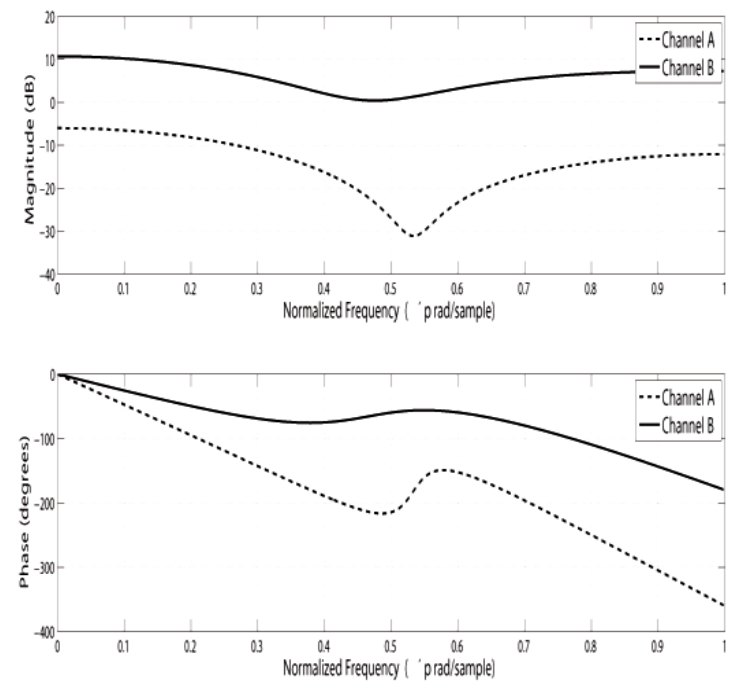

Fig. 1. Frequency responses of FIR channels. 


\section{Simulation Results}

In this section, performance of the proposed algorithm is compared with the CMB-RLS algorithm in a typical DMT environment. The system has FFT size $m=52$, the CP length $v=4, \mu_{1}=\lambda=0.7, \mu_{2}=1, \mathrm{SNR}=30 \mathrm{~dB}$ and constellation was set to 4-QAM. The performance is evaluated by averaged mean-square-error (MSE) per subchannel err $=\sum_{i \in U}\left\|X_{i}-\hat{X}_{i}\right\| /|U|$, where $U$ is the set of indexes corresponding to the $U$ used subchannels and $|U|$ is the number of all the used subchannels. The transmit power of all used subchannels is same (i.e., $\sigma_{i}{ }^{2}=\sigma^{2}$ ). The two static finite impulse response (FIR) test channels used in simulation example are listed in Table II, whereas, corresponding frequency responses are shown in Fig. 1. Only the first DMT symbol was sent as pure training sequence to identify the initial channel $\mathbf{h}_{0}$ for fast convergence.

The learning curves of the two algorithms are plotted in Fig. 2. Initially, the channel is having impulse response of Channel A, which remains unchanged for the first 250 data blocks. At data block 251, the channel impulse response switches to Channel B. It can be seen from Fig. 2 that both the algorithms are able to converge and track the channel variation. Notice that initial convergence of the extended QRD-RLS algorithm is similar to the CMB-RLS algorithm, however, its estimation quality improves with time and the algorithm outperforms CMB-RLS approach in terms of the steady state error performance.

\section{CONCLUSION}

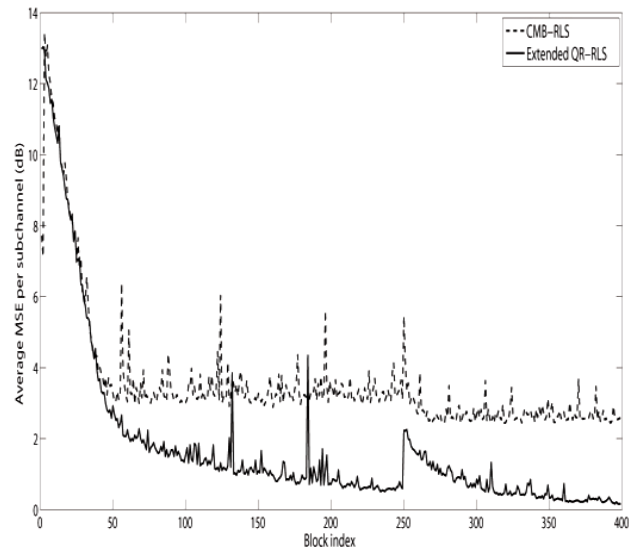

Fig. 2. Average MSE per subchannel learning curves of CMB-RLS and extended QRD-RLS algorithms when tracking channel A and B.

This paper has presented a new CP based extended QRD-RLS algorithm using block Householder transformations for channel tracking in multicarrier systems. Compared to the CP based CMB-RLS algorithm described in [7], which requires $O\left(r^{3}\right)$ computations in the channel estimation part, the proposed algorithm involves $O\left(r^{2}\right)$ computations. Simulation results show that the new algorithm shows better steady state performance than its counterpart in a floating-point representation. The algorithm can be efficiently executed on systolic arrays. It is therefore very promising for very-large scale integration (VLSI) systolic implementation.

\section{REFERENCES}

[1] Z. Wang and G. B. Giannakis, "Wireless multicarrier communications", IEEE Signal Processing Magazine, vol. 17, no. 3 , pp. $29-48,2000$.

[2] "IEEE Part II: Wireless LAN medium access control (MAC) and physical layer (PHY) specifications: High speed physical layer in 5 GHz band," IEEE Std, 1999.

[3] K. V. Acker, G. Leus, M. Moonen, O. V. D. Wiel, and T. Pollet, "Per ton equalization for DMT-based systems," IEEE Transactions on Communications, vol. 49, no. 1, pp. 109-119, 2001.

[4] P. J. W. Melsa, R. C. Younce, and C. E. Rohrs, "Impulse response shortening for discrete multitone transceivers," IEEE Transactions on Communications, vol. 44, no. 12, pp. $1662-1672,1996$.

[5] P. S. Chow, J. M. Cioffi, and J. A. C. Bingham, "A practical discrete multitone transceiver loading algorithm for data transmission over spectrally shaped channels," IEEE Transactions on Communications, vol. 43, pp. 773-775, 1995.

[6] J. S. Chow, J. C. Tu, and J. M. Cioffi, “A discrete multitone transceiver system for HDSL applications," IEEE Journal on Selected Areas in Communications, vol. 9, no. 6, pp. $895-908,1991$.

[7] X. Wang and K. J. R. Liu, "Adaptive channel estimation using cyclic prefix in multicarrier modulation system," IEEE Communications Letters, vol. 3, no. 10, pp. 291-293, 1999.

[8] K. J. R. Liu, S. F. Hsieh, and K. Yao, "Systolic block Householder transformation for RLS algorithm with two-level pipelined implementation," IEEE Transactions on Signal Processing, vol. 40, no. 4, pp. 946-958, 1992.

[9] B. Yang and J. Bohme, "Rotation-based RLS algorithms: unified derivations, numerical properties, and parallel implementations", IEEE Transactions on Signal Processing, vol. 40, no. 5, pp. $1151-1167$, 1992.

[10] G. H. Golub and C. F. V. Loan, Matrix Computations, 3rd Edition, the Johns Hopkins University Press, 1996.

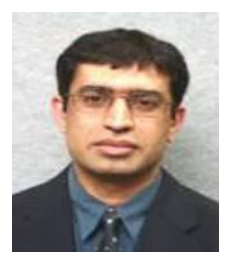

Hassan Ali was born in Hyderabad, Pakistan in 1970. He received the B.E. degree in electrical engineering from the Mehran University of Engineering and Technology (MUET), Pakistan, in 1994 and the Ph.D. degree, from the University of Melbourne, Parkville, Australia, in 2003. From 1994 to 1999 , he was a Project Officer with the Private Power and Infrastructure Board (PPIB), Islamabad, Pakistan. From 2000 to 2003, he was an Overseas Postgraduate Research Scholar, with the Signals and Systems Research Group and ARC Special Research Center for Ultra-Broadband Information Networks (CUBIN), University of Melbourne. From September 2002 to September 2003, he was a Lecturer of Communications and Signal Processing with Curtin University of Technology, Perth, WA. From 2004 to April 2008, he was an Assistant Professor with The University of Nottingham, Malaysia Campus. $\mathrm{He}$ is currently Lecturer of Electrical Engineering in the Faculty of Engineering and Built Environment, University of Newcastle, Australia (Singapore Campus). His current research includes adaptive and array signal processing, blind channel identification and equalization for wireless broadband systems, PAR reduction in OFDM, and single and multiuser detection for CDMA systems. In 2000, Dr. Hassan was awarded Overseas Postgraduate Research Scholarship (OPRS) by the Australian Government and Melbourne International Research Scholarship (MIRS) by the University of Melbourne. During 2001 and 2002 he received the Department of Electrical and Electronic Engineering, University of Melbourne, Postgraduate Studentship. In 2001, he was awarded the Excellent Graduates Awards: the Gold Medal and the Silver Medal by MUET. 